\title{
EXISTENCE OF SOLUTIONS OF EXTREMAL PROBLEMS IN $H^{\prime}$
}

\author{
by TAKAHIKO NAKAZI*
}

(Received 14th April 1989)

An essentially bounded function on the unit circle gives a continuous linear functional on the Hardy space $H^{1}$. In this paper we study when there exists at least one function which attains its norm. We apply the results to an interpolation problem, Hankel operators and a characterization of exposed points of the closed unit ball of $H^{1}$.

1980 Mathematics subject classification: Primary 30 D 55, 47 B 35; Secondary 46 J 15.

\section{Introduction}

Let $H^{p}$ be the usual Hardy spaces on the unit circle $T$ for $p \geqq 1$. If $\phi \in L^{\infty}$, we denote by $T_{\phi}$ the functional defined on $H^{1}$ by

$$
T_{\phi}(f)=\int_{-\pi}^{\pi} f\left(e^{i \theta}\right) \phi\left(e^{i \theta}\right) d \theta / 2 \pi
$$

Let $S_{\phi}$ be the set of functions in $H^{1}$ which satisfy $T_{\phi}(f)=\left\|T_{\phi}\right\|$ and $\|f\|_{1} \leqq 1$. We define $\rho(\phi)$ to be the set of all complex numbers $s$ for which $S_{\phi-s}$ is nonempty. If $\phi \in C$, then $T_{\phi-s}$ is weak-* continuous on $H^{1}$ for any $s \in \mathbb{C}$ and hence $S_{\phi-s}$ is nonempty, that is, $\rho(\phi)=\mathbb{C}$ where $C$ denotes the space of continuous functions on the unit circle and $\mathbb{C}$ is the set of all complex numbers. $S_{\phi}$ can be empty for some $\phi \in L^{\infty}$ and hence $\rho(\phi) \neq \mathbb{C}$. Many mathematicians have studied the structure of $S_{\phi}$ when $S_{\phi}$ is nonempty (see [1], [2, Chapter 8], [3, Chapter IV], [4], [9] and [10]). Rogosinski and Shapiro, and Caughran gave the examples of $\phi$ with $0 \notin \rho(\phi)$ (see [2, Chapter 8]). However $\rho(\phi)$ has not been studied systematically. In this paper we describe $\rho(\phi)$ in general and apply our results to concrete $\phi$.

In Section 2, we show that $\rho(\phi)=\mathbb{C}$ if $\left\|\phi+H^{\infty}\right\| \neq\left\|\phi+H^{\infty}+C\right\|$. In Section 3, we prove that $\rho(\phi) \supset \mathbb{C} \backslash E(\phi)$ where $E(\phi)=\left\{f(0):\|\phi-f\|_{\infty}=\left\|\phi+H^{\infty}\right\|\right\}$. In Section 4, using a well known theorem of Adamyan, Arov and Krein (cf. [3, Chapter IV, Theorem 5. 3]) it is shown that $\rho(\phi) \subset \mathbb{C} \backslash E(\phi)^{\circ}$ if $\rho(\phi) \neq \mathcal{C}$. In Section $5, E(\phi)$ is described, in fact, it is a closed disc. For special $\phi$, an explicit description is given. In Sections 6 and 7 we consider $\rho(\phi)$ in case $\phi$ is a quotient of two inner functions. In Section 8 we give

*This research was partially supported by Grant-in-Aid for Scientific Research, Ministry of Education. 
applications to a minimal interpolation problems, Hankel operators and a characterization of exposed points of ball $\left(H^{1}\right)$, the closed unit ball of $H^{1}$.

2. $\rho(\phi)=\mathbb{C}$ if $\left\|\phi+H^{\infty}\right\| \neq\left\|\phi+H^{\infty}+C\right\|$

We denote the maximal ideal space of $L^{\infty}$ by $X$ and the Gelfand transform of the function $\phi$ in $L^{\infty}$ by $\hat{\phi}$. Then $L^{\infty}$ is isometrically isomorphic to the algebra $C(X)$ of all continuous functions on $X$, that is, $L^{\infty} \cong \widehat{L}^{\infty}=C(X)$. Hence $\left(L^{\infty}\right)^{*} \cong M(X)$, where $M(X)$ is the set of all complex regular Borel measures on $X$. For each $\bar{\phi} \in C(X)$, if we assign the number $\int_{-\pi}^{\pi} \phi\left(e^{i \theta}\right) d \theta / 2 \pi$ to it then there exists a probability measure $m$ on $X$ such that $\int_{-\pi}^{\pi} \phi d \theta / 2 \pi=\int_{X} \hat{\phi} d m$ for all $\phi$. Let $M^{s}$ be the set of all complex singular measures with respect to $m$, then $M(X)=L^{1}(m) \oplus M^{s}$. $L^{1}$ is canonically embedded into the bidual $\left(L^{\infty}\right)^{*}$ and $L^{1} \cong L^{1}(m)$. If we set

$$
\begin{aligned}
\mathscr{H} & =\left(\hat{z} \hat{H}^{\infty}\right)^{\perp} \cap M(X) \\
& =\left\{v \in M(X): \int_{X} \hat{f} d v=0 \text { for all } f \in z H^{\infty}\right\},
\end{aligned}
$$

then $\mathscr{H} \cong\left(z H^{\infty}\right)^{\perp} \cap\left(L^{\infty}\right)^{*}=\left(L^{\infty} / z H^{\infty}\right)^{*}=\left(H^{1}\right)^{* *}$. By the F. and M. Riesz theorem for $H^{\infty}$ (cf. [5, p. 186], $\mathscr{H}=\mathscr{H} \cap L^{1}(m) \oplus \mathscr{H} \cap M^{s} . H^{1}$ is canonically embedded into the bidual $\left(H^{1}\right)^{* *}$ and $H^{1} \cong \mathscr{H} \cap L^{1}(m)$.

If $\phi \in L^{\infty}$, we denote by $\mathscr{T}_{\phi}$ the functional defined on $\mathscr{H}$ by

$$
\mathscr{T}_{\phi}(v)=\int_{X} \hat{\phi} d v
$$

The norm of $\mathscr{T}_{\phi}$ is $\left\|\mathscr{T}_{\phi}\right\|=\sup \left\{\left|\mathscr{T}_{\phi}(v)\right|: v \in \mathscr{P}\right\}$ and let $\mathscr{S}_{\phi}$ denote the set of all $v \in \mathscr{S}$ for

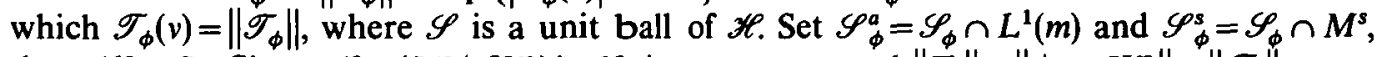
then $\mathscr{S}_{\phi}^{a} \cong S_{\phi}$. Since $\mathscr{H} \cong\left(L^{\infty} / z H^{\infty}\right)^{*}, \mathscr{S}_{\phi}$ is not empty and $\left\|T_{\phi}\right\|=\left\|\phi+z H^{\infty}\right\|=\left\|\mathscr{T}_{\phi}\right\|$.

Lemma 1. If $\phi \in L^{\infty}$, then

$$
\max \left\{\left|\mathscr{T}_{\phi}(v)\right|: v \in \mathscr{S} \cap M^{s}\right\}=\left\|\phi+H^{\infty}+C\right\|
$$

Proof. If $v \in \mathscr{S} \cap M^{s}$ then the $v$ annihilates $\hat{C}$ by the $\mathrm{F}$. and $\mathrm{M}$. Riesz theorem for $H^{\infty}$ (cf. [5, p. 186]) and so $\sup \left|\mathscr{T}_{\phi}(v)\right|=\left\|\phi+H^{\infty}+C\right\|$. If $v_{n} \in S \cap M^{2}$ and $\left|\mathscr{T}_{\phi}\left(v_{n}\right)\right| \rightarrow$ $\sup \left|\mathscr{T}_{\phi}(v)\right|$ as $n \rightarrow \infty$, there exists $v_{\infty} \in \mathscr{S}$ such that $\left|\mathscr{T}_{\phi}\left(v_{\infty}\right)\right|=\sup \left|\mathscr{T}_{\phi}(v)\right|$ and $v_{n j} \rightarrow v_{\infty}$ in the weak-* topology of $\mathscr{H}$, where $\left\{v_{n j}\right\}$ is a subsequence of $\left\{v_{n}\right\}$. Since $v_{n j}$ annihilates $\hat{C}$, $v_{\infty}$ annihilates $\hat{C}$, too, and so $v_{\infty} \in \mathscr{S} \cap M^{s}$.

Proposition 1. Let $\phi \in L^{\infty}$. Then

(1) $\mathscr{S}_{\phi}$ is nonempty; 
(2) $\mathscr{S}_{\phi}=\left\{\gamma \mathscr{S}_{\phi}^{a}+(1-\gamma) \mathscr{S}_{\phi}^{s}: 0 \leqq \gamma \leqq 1\right\}$

(3) $\mathscr{S}_{\phi}=\mathscr{S}_{\phi}^{a}$ if and only if $\left\|\phi+z H^{\infty}\right\| \supsetneqq\left\|\phi+H^{\infty}+C\right\|$.

Proof. (1) was proved already. (2) It is clear that $\mathscr{S}_{\phi} \supseteq\left\{\gamma \mathscr{S}_{\phi}^{a}+(1-\gamma) \mathscr{S}_{\phi}^{s}\right\}$. If $v \in \mathscr{S}_{\phi}$, we can write $v=k d m+v^{s}$ for $k \in \mathscr{H} \cap L^{1}(m)$ and $v^{s} \in \mathscr{H} \cap M^{s}$ and then $\log |k| \in L^{1}(m)$. For $\mathscr{H}=\mathscr{H} \cap L^{1}(m) \oplus \mathscr{H} \cap M^{s}$ and $\mathscr{H} \cap L^{1}(m) \cong H^{1}$. We can show that $\hat{\psi} v=|v|$ a.e. $|v|$ for the extremal kernel $\psi$ of $\phi$. Hence $\hat{\psi} k=|k|$ a.e. $m$ and $\hat{\psi} v^{s}=\left|v^{s}\right|$ a.e. $\left|v^{s}\right|$. Since $k \in \mathscr{H} \cap L^{1}(m)$ and $v^{s} \in \mathscr{H} \cap M^{s}, k /\|k\|_{1}$ belongs to $\mathscr{S}_{\phi}^{a}$ and $v^{s} /\left\|v^{s}\right\|$ belongs to $\mathscr{S}_{\phi}^{s}$ and $\|k\|_{1}+\left\|v^{s}\right\|=\|v\|=$ 1. Thus $v \in\left\{\gamma \mathscr{S}_{\phi}^{a}+(1-\gamma) \mathscr{S}_{\phi}^{s}: 0 \leqq \gamma \leqq 1\right\}$.

(3) By (1), $\mathscr{S}_{\phi}^{s}$ is empty if and only if $\mathscr{S}_{\phi}=\mathscr{S}_{\phi}^{a}$. This and Lemma 1 imply (3).

It is interesting to find the condition on $\phi$ which implies that $\mathscr{S}_{\phi}=\mathscr{S}_{\phi}^{\mathrm{s}}$. For $\mathscr{S}_{\phi}=\mathscr{S}_{\phi}^{\text {s }}$ if and only if $\mathscr{S}_{\phi}^{a}$ is empty, by Proposition 1. The following is the first result about $\rho(\phi)$.

Proposition 2. Let $\phi \in L^{\infty}$. Then the following (1) and (2) are valid.

(1) If $\left\|\phi+z H^{\infty}\right\| \geqq\left\|\phi+H^{\infty}+C\right\|$ then $\rho(\phi) \ni 0$.

(2) If $\left\|\phi+H^{\infty}\right\| \gtreqless\left\|\phi+H^{\infty}+C\right\|$ then $\rho(\phi)=\varnothing$.

Proof. (1) is clear by (3) of Proposition 1 because $\mathscr{S}_{\phi}^{a} \cong \mathscr{S}_{a}$. (2) For any $s \in \mathbb{C}$, $\left\|\phi-s+z H^{\infty}\right\| \geqq\left\|\phi+H^{\infty}\right\| \supsetneqq\left\|\phi+H^{\infty}+C\right\|$ and hence (1) implies that $s \in \rho(\phi)$.

Proposition 2 is well known and it implies that if $\phi \in H^{\infty}+C$ then $\rho(\phi)=\mathbb{C}$ (see [1]).

3. $\rho(\phi) \supset \mathbb{C} \backslash E(\phi)$

Recall that $\rho(\phi)$ and $E(\phi)$ were defined in the Introduction.

Lemma 2. If $\phi \in L^{\infty}$, then for any $f \in H^{\infty}$ and any $a \in \mathbb{C}$

$$
\rho(a \phi+f)=f(0)+a \rho(\phi) .
$$

Proof. $S_{a \phi+f}=S_{a \phi+f(0)}$, and when $a \neq 0, s \in \rho(a \phi+f(0))$ if and only if $(s-f(0)) /$ $a \in \rho(\phi)$. This implies the lemma.

Theorem 3. Let $\phi \in L^{\infty}$. Then the following (1)-(3) are valid.

(1) $\rho(\phi) \supseteq \int \phi d \theta / 2 \pi+\left\{s \in \mathbb{C}:|s|>\left\|\phi+H^{\infty}\right\|\right\}$.

(2) $\rho(\phi) \supset \mathbb{C} \backslash E(\phi)$.

(3) If $E(\phi)$ is a single point s then $\rho(\phi)=\mathbb{C} \backslash\{s\}$ or $\rho(\phi)=\mathbb{C}$.

Proof. (1) If $|s| \supsetneqq\left|\int \phi d \theta / 2 \pi\right|+\left\|\phi+H^{\infty}\right\|$ then

$$
|| \phi-s+z H^{\infty}|| \geqq\left|\int \phi-s d \theta / 2 \pi\right| \geqq|s|-\left|\int \phi d \theta / 2 \pi\right|
$$




$$
\gtreqless\left\|\phi+H^{\infty}\right\|
$$

and hence by (1) of Proposition $20 \in \rho(\phi-s)$. Thus

$$
\rho(\phi) \supseteq\left\{s \in \mathbb{C}:|s|>\left|\int \phi d \theta / 2 \pi\right|+\left\|\phi+H^{\infty}\right\|\right.
$$

and hence

$$
\rho\left(\phi-\int \phi d \theta / 2 \pi\right) \supseteq\left\{s \in \mathbb{C}:|s|>\left\|\phi+H^{\infty}\right\|\right\}
$$

because $\int\left(\phi-\int \phi d \theta / 2 \pi\right) d \theta / 2 \pi=0$. Now Lemma 2 implies (1).

(2) If $s \in \mathbb{C} \backslash E(\phi)$ then there exists $g \in H^{\infty}$ such that $\left\|\phi-s+z H^{\infty}\right\|=\|\phi-s+z g\|_{\infty}$ and

$$
\|\phi-s+z g\|_{\infty} \supsetneqq\left\|\phi+H^{\infty}\right\| .
$$

By (1) of Proposition 2, $s \in \rho(\phi)$ and hence $\rho(\phi) \supset \mathbb{C} \backslash E(\phi)$. (3) is clear by (2).

(2) of Theorem 3 is essential in this paper. The following theorem, which is its corollary, is a little surprising. For if $\rho(\phi) \neq \mathbb{C}$ then for any $s \in \rho(\phi), S_{\phi-s}$ consists of one element.

Theorem 4. If $\phi \in L^{\infty}$ and $S_{\phi}$ contains at least two functions then $\rho(\phi)=\mathbb{C}$.

Proof. Since $0 \in \rho(\phi)$, by (3) of Theorem 3, it is sufficient to show that $E(\phi)$ is a single point 0 . Suppose $f \in H^{\infty}$ and $\|\phi+f\|_{\infty}=\left\|\phi+H^{\infty}\right\|$. We will show that if $\left\|\phi+z H^{\infty}\right\|=\|\phi\|_{\infty}$ then $f=0$ a.e.. By hypothesis and Theorem 9 in [1], $S_{\phi} \ni z h$ for some $h \in H^{1}$. Therefore $\left\|T_{\phi}\right\|=\left\|T_{z \phi}\right\|$ and hence $\left\|\phi+z H^{\infty}\right\|=\left\|\phi+H^{\infty}\right\|$. Since $S_{\phi} \ni z h, S_{z \phi}$ is nonempty and hence there exists a unique $g \in H^{\infty}$ such that

$$
\begin{aligned}
\|z \phi+z g\|_{\infty} & =\left\|z \phi+z H^{\infty}\right\|=\left\|\phi+H^{\infty}\right\| \\
& =\left\|\phi+z H^{\infty}\right\|=\|\phi\|_{\infty}=\|z \phi\|_{\infty}
\end{aligned}
$$

and hence $g=0$ a.e.. Now $\|z \phi+z f\|_{\infty}=\left\|z \phi+z H^{\infty}\right\|$ and hence $f=0$ a.e.. If $\left\|\phi+z H^{\infty}\right\|$ $\neq\|\phi\|_{\infty}$ then by Theorem 8.1 in [2] there exists $\psi \in L^{\infty}$ such that

$$
\left\|\psi+z H^{\infty}\right\|=\|\psi\|_{\infty} \quad \text { and } \quad \psi=\phi+z k
$$

for some nonzero $k \in H^{\infty}$. By Lemma $2, E(\psi)=E(\phi)$ and hence from what was shown above $E(\phi)=\{0\}$ follows.

The following lemma due to P. Koosis (cf. [3, Chapter IV, Lemma 5.4]) will be used several times in this paper.

Lemma 3. If $\phi \in L^{\infty}$ with $|\phi|=1$ a.e. and there is $k \in H^{\infty}, k \neq 0$, such that $\|\phi-k\|_{\infty} \leqq 1$, then there exists an outer function $g \in H^{1},\|g\|_{1}=1$, such that $\phi=g /|g|$ a.e.. 
Corollary 1. Let $\phi \in L^{\infty}$. Then the following (1)-(4) are valid.

(1) If $\phi+H^{\infty}$ is an extreme point of the ball $\left(L^{\infty} / H^{\infty}\right)$ then $\rho(\phi)=\mathbb{C} \backslash\{0\}$ or $\rho(\phi)=\mathbb{C}$.

(2) If $\bar{\phi}$ is an inner function then $\rho(\phi)=\mathbb{C}$.

(3) If $\phi=2 \chi_{F}-1$ and $0<d \theta(F)<2 \pi$ then $\rho(\phi)=\mathbb{C} \backslash\{0\}$.

(4) If $\phi=|f| / f$ for some nonzero $f \in H^{1}$ with $f^{-1} \notin H^{1}$ then $\rho(\phi)=\mathbb{C}$.

Proof. (1) By Exercise 17 in [3, Chapter IV], if $\phi+H^{\infty}$ is an extreme point then $\|\phi+f\|_{\infty}>1$ for all $f \in H^{\infty}$ with $f \neq 0$. Hence $E(\phi)=\{0\}$. (3) of Theorem 3 implies (1). (2) If $\bar{\phi}$ is a finite Blaschke product then by (2) of Theorem $2 \rho(\phi)=\mathbb{C}$. If $\phi$ is not so then $S_{\phi}$ contains at least two functions (see Lemma 2 in [10]) and hence by Theorem 4 $\rho(\phi)=\mathscr{C}$. (3) follows immediately from Example in [7, p. 198]. (4) By Lemma 3 if there exists a nonzero $g \in H^{\infty}$ such that $\|\phi+g\|_{\infty} \leqq 1$ then there exists a nonzero $h \in H^{1}$ and $\phi=h /|h|$. Therefore $h f$ is nonnegative and hence constant because $H^{1 / 2}$ does not contain nonconstant nonnegative functions (cf. [3, Chapter II, Exercise 13]). This contradicts the fact that $f^{-1} \notin H^{1}$ and hence $E(\phi)=\{0\}$. (3) of Theorem 3 implies $\rho(\phi)=\mathbb{C}$ because $S_{\phi}$ is nonempty.

\section{4. $\rho(\phi) \subset \mathbb{C} \backslash E(\phi)^{0}$}

In Section 3 we showed that if $E(\phi)$ is a single point and $\rho(\phi) \neq \mathbb{C}$ then $\rho(\phi)=\mathbb{C} \backslash E(\phi)$. We can ask whether or not this is true for arbitrary $E(\phi)$. However we can show that if $\rho(\phi) \neq \mathbb{C}$ then $\rho(\phi) \subset \mathbb{C} \backslash E(\phi)^{0}$ where $E(\phi)^{0}$ denotes the interior of $E(\phi)$.

For any nonzero $h \in H^{1}$, define $Q_{h} \in H^{\infty}$ by

$$
\frac{1+Q_{h}(z)}{1-Q_{h}(z)}=\frac{1}{2 \pi} \int \frac{e^{i t}+z}{e^{i t}-z}\left|h\left(e^{i \eta}\right)\right| d t .
$$

For any $\phi \in L^{\infty}$, put

$$
K_{\phi}=\left\{f \in H^{\infty}:\|\phi-f\|_{\infty} \leqq 1\right\} .
$$

The following Lemma 4 is Exercise 18 in [3, Chapter IV] which is essentially due to Adamyan, Arov and Krein (cf. [3, Chapter IV, Theorem 5.3]).

Lemma 4. Let $\phi=h /|h|$ for some nonzero $h \in H^{1} . h$ is an exposed point of the ball $\left(H^{1}\right)$ if and only if

$$
K_{\phi}=\left\{\frac{h\left(1-Q_{h}\right)(1-w)}{1-Q_{h} w}: w \in H^{\infty} \text { and }\|w\|_{\infty} \leqq 1\right\}
$$

Lemma 5. If $\phi=h /|h|$ and $h$ is an exposed point of the ball $\left(H^{1}\right)$ then 


$$
K_{\phi}(0)=\{z \in \mathbb{C}:|z-h(0)| \leqq|h(0)|\}
$$

The proof is clear.

Theorem 5. Let $\phi \in L^{\infty}$. If $\rho(\phi) \neq \mathbb{C}$ then $\rho(\phi) \subset \mathbb{C} \backslash E(\phi)^{0}$.

Proof. We can assume that $E(\phi)$ has a nonempty interior. Moreover we may assume $\left\|\phi+H^{\infty}\right\|=1$. If $s \in E(\phi)^{0} \cap \rho(\phi)$ then by [1, p. 479] there exist $f \in H^{1}$ with $\|f\|_{1}=1$ and $k \in H^{\infty}$ such that

$$
\phi-s-z k=|f| / f
$$

If $k$ is. a nonzero function or $s \neq 0$ then by Lemma $3, f^{-1}$ belongs to $H^{1}$. If $k=0$ a.e. and $s=0$ then $\phi=|f| / f$. If $f^{-1} \notin H^{1}$, this contradicts the hypothesis by (4) of Corollary 1 and hence $f^{-1}$ belongs to $H^{1}$. Then $f^{-1} /\left\|f^{-1}\right\|$ is an exposed point of ball $\left(H^{1}\right)$ (see [9, Proposition 5]) and hence by Lemma 5

$$
E\left(\frac{|f|}{f}\right)=\left\{z \in \mathbb{C}:\left|z-f^{-1}(0)\right| \leqq\left|f^{-1}(0)\right|\right\}
$$

because $\left\|\phi+H^{\infty}\right\|=1$. But

$$
E\left(\frac{\lfloor f\rfloor}{f}\right)=E(\phi-s)=E(\phi)-s
$$

and hence $E(|f| / f)$ contains 0 as an interior because $s \in E(\phi)^{0}$. This contradiction implies that $E(\phi)^{0} \cap \rho(\phi)=\emptyset$.

\section{Description of $E(\phi)$}

In the previous sections, we showed that

$$
\mathbb{C} \backslash E(\phi) \subset \rho(\phi) \subset \boldsymbol{C} \backslash E(\phi)^{0} .
$$

Therefore it will be useful to describe $E(\phi)$. These are corollaries of a powerful result of Adamyan, Arov and Krein (cf. [3, Chapter IV, Theorem 5.3]).

Let $\phi \in L^{\infty}$ and $\phi \notin H^{\infty}$. If $E(\phi)$ is not a single point there exists a unique outer function $F \in H^{1}$ with $F /|F| \in \phi / a+H^{\infty},\|F\|_{1}=1$ and

$$
\operatorname{Re} \int \frac{F}{|F|} d \theta / 2 \pi=\sup \left\{\operatorname{Re} \int\left(\frac{\phi}{a}-k\right) d \theta / 2 \pi:\left\|\frac{\phi}{a}-k\right\|_{\infty}=1\right\}
$$

where $a=\left\|\phi+H^{\infty}\right\|$ (see [3, Chapter IV, Theorem 5.3]). 
Proposition 6. Let $\phi \in L^{\infty}$ with $\phi \notin H^{\infty}$ and $a=\left\|\phi+H^{\infty}\right\|$. If $E(\phi)$ is not a single point then for the $F$ defined above

$$
E(\phi)=a\left\{z \in \mathbb{C}:\left|z-z_{0}\right| \leqq|F(0)|\right\}
$$

where

$$
z_{0}=\int \frac{\phi}{a} d \theta / 2 \pi-\int \frac{F}{|F|} d \theta / 2 \pi+F(0)
$$

In particular $E(\phi)$ is a closed disc.

Proof. Since $\left\|\phi / a+H^{\infty}\right\|=1, E(\phi / a)=K_{\phi / a}(0)$. By Theorem 5.3 in [3, Chapter IV],

$$
K_{\phi / a}=\left\{f=\frac{\phi}{a}-\frac{F}{|F|}+\frac{F\left(1-Q_{F}\right)(1-w)}{1-Q_{F} w}: w \in H^{\infty},\|w\|_{\infty} \leqq 1\right\}
$$

Hence

$$
E(\phi / a)=\left\{\int \frac{\phi}{a} d \theta / 2 \pi-\int \frac{F}{|F|} d \theta / 2 \pi+F(0)(1-w(0)): w \in H^{\infty},\|w\|_{\infty} \leqq 1\right\} .
$$

This implies the proposition.

We will concentrate on unimodular functions, that is, $\phi \in L^{\infty}$ and $|\phi|=1$ a.e.. Then we can describe $E(\phi)$ more exactly than Proposition 6.

Lemma 6. Let $\phi=f /|f|$ for some nonzero $f \in H^{1}$. Then

$$
K_{\phi}=\left\{\frac{g\left(1-Q_{g}\right)(1-w)}{1-Q_{g} w}: w \in H^{\infty},\|w\|_{\infty} \leqq 1 \text { and } g \in S_{\bar{\phi}}\right\}
$$

Proof. By Lemma 5.5 in [3, Chapter IV],

$$
K_{\phi} \supseteq\left\{\frac{g\left(1-Q_{\theta}\right)(1-w)}{1-Q_{g} w}: w \in H^{\infty},\|w\|_{\infty} \leqq 1 \text { and } g \in S_{\bar{\phi}}\right\}
$$

For the reverse inclusion, if $\|\phi-k\|_{\infty} \leqq 1$, set $\alpha=\arg \phi k$ and $\psi=e^{\tilde{\alpha}-i \alpha}$; then $\psi k \in H^{1}$ and

$$
\phi=g /|g| \text { and } g=\psi k /\|\psi k\|_{1}
$$

(see [3, Chapter IV, Lemma 5.4]). This implies $g \in S_{\bar{\phi}}$ and by the proof of Theorem 5.3 in [3, Chapter IV], 


$$
k=\frac{g\left(1-Q_{g}\right)(1-w)}{1-Q_{g} w}
$$

for some $w \in H^{\infty}$.

Proposition 7. Let $\phi \in L^{\infty}$ and $|\phi|=1$ a.e..

(1) If $\phi$ is not of the form $\phi=f /|f|$ for some nonzero $f \in H^{1}$, then $E(\phi)=\{0\}$.

(2) If $\phi=f /|f|$ for some nonzero $f \in H^{1}$ with $\|f\|_{1}=1$, then

$$
E(\phi) \subseteq\left\{z \in \mathbb{C}:|z-g(0)| \leqq|g(0)|, g \in S_{\bar{\phi}}\right\}
$$

If $\left\|\phi+H^{\infty}\right\|=1$ then

$$
E(\phi)=\left\{z \in \mathbb{C}:|z-g(0)| \leqq|g(0)|, g \in S_{\bar{\phi}}\right\}
$$

and hence $E(\phi)$ is not a single point.

Proof. (1) From Lemma $3, E(\phi)=\{0\}$ obviously follows. (2) Evaluate $K_{\phi}$ in Lemma 6 at $z=0$; then it contains $E(\phi)$ and if $\left\|\phi+H^{\infty}\right\|=1$ then it coincides with $E(\phi)$. This implies (2).

\section{6. $\rho(\phi)$ for special symbols $\phi$}

Let $q$ be an inner function and $k$ be in $H^{\infty}$. In this section for a special $\phi \in L^{\infty}$ such that $\phi=\bar{q} k$ we will study $\rho(\phi)$.

Proposition 8. Let $q$ be an inner function and $k \in H^{\infty}$. If $\phi=\bar{q} k,\left\|T_{\phi}\right\|=\alpha>0$ and $0 \in \rho(\phi)$ then there exists an inner function $q_{0}$ such that $\bar{q} k-\alpha \bar{q} q_{0}$ is in $z H^{\infty}$. Then $\alpha \bar{q} q_{0}$ is an extremal kernel, that is, $\left\|\alpha \bar{q} q_{0}+z H^{\infty}\right\|=\alpha$. In particular, $\rho(\bar{q} k)=\alpha \rho(\bar{q} k)$.

Proof. If $f \in S_{\phi}$ then $f \in S_{\phi / \alpha}$ and $\left\|T_{\phi / \alpha}\right\|=1$. Hence there exists a function $g \in H^{\infty}$ such that $\bar{q} k / \alpha+z g=|f| / f$. Let $q_{0}=k / \alpha+z q g$; then $q_{0}$ is an inner function and $\alpha \bar{q} q_{0}=\bar{q} k+z \alpha g$. This implies the proposition.

For each function $f$ in $H^{1}$, sing $f$ denotes the set of the unit circle on which $f$ cannot be analytically extended. Let $q$ and $q_{0}$ be inner functions. $q_{0}$ is called the maximum multiplier of a nonzero function $h$ in $H^{2} \ominus q z H^{2}$ if $q_{0} h \in H^{2} \ominus q z H^{2}$ and $q_{1} h \in H^{2} \ominus q z H^{2}$ for some inner function $q_{1}$ implies that $q_{0} \bar{q}_{1} \in H^{\infty}$. Since $q \bar{h}$ is in $H^{2}, q_{0}$ can be obtained as the inner part of $q \bar{h}$.

Theorem 9. Let $q$ and $q_{0}$ be inner functions, and suppose $\phi=\bar{q} q_{0}$.

(1) $0 \in \rho(\phi)$ and $\phi$ is an extremal kernel if and only if there exists a nonzero function $f$ in $H^{2} \ominus z q H^{2}$ such that $\bar{q}_{0} f$ is in $H^{2}$. 
(2) If $q_{0}$ is not the maximum multiplier of a nonzero function $f$ in $H^{2} \ominus z q H^{2}$ but $\bar{q}_{0} f \in H^{2}$, then $\rho(\phi)=\mathbb{C}$.

(3) If $0 \in \rho(\phi)$ and $\phi$ is an extremal function then sing $q \supset$ sing $q_{0}$.

(4) If (sing $q) \cap\left\{T \backslash\right.$ sing $\left.q_{0}\right\}$ is nonempty then $\rho(\phi)=\mathbb{C} \backslash\{0\}$ or $\rho(\phi)=\mathbb{C}$.

(5) Let (sing q) $\cap\left(\operatorname{sing} q_{0}\right)$ be empty. If $q_{0}$ is a finite Blaschke product then $\rho(\phi)=\mathbb{C}$ and if $q_{0}$ is not so then $\rho(\phi)=\varnothing \backslash\{0\}$.

Proof. (1) If $f \in H^{2} \ominus q z H^{2}$ and $f=q_{0} h$ for some $h \in H^{2}$ then $q \bar{q}_{0} \bar{h} \in H^{2}$. Hence $q \bar{q}_{0}|h|^{2} \in H^{1}$ and this implies that $0 \in \rho(\phi)$ and $\phi$ is an extremal kernel. Conversely if $0 \in \rho(\phi)$ and $\phi$ is an extremal kernel then there exists an outer function $h$ such that $\bar{z} \bar{q} q_{0} h=\bar{z} \bar{h}$. Hence $q_{0} h$ is orthogonal to $z q H^{2}, f=q_{0} h$ is a desired function.

(2) If $\bar{q}_{0} f \in H^{2}$ but $q_{0}$ is not the maximum multiplier then there exists an inner function $q_{1}$ such that $\bar{q}_{1} \bar{q}_{0} f \in H^{2}$. By (1) $\bar{q} q_{0} q_{1}$ is the extremal kernel and $S_{\bar{q} q 0 q_{1}}$ is nonempty. Hence $S_{\phi}$ has at least two functions. For $S_{\phi} \supsetneq q_{1} S_{\bar{q} q \circ q_{1}}$ because $S_{\phi}$ contains always an outer function. By Theorem $4 \rho(\phi)=\mathbb{C}$.

(3) By (1) there exists a nonzero function $f \in H^{2} \ominus q z H^{2}$ and $f=q_{0} h$ for some $h \in H^{2}$. It is known that sing $f \subset \operatorname{sing} q$. By Lemma 4 in [7] sing $q_{0} \subset \operatorname{sing} q$.

(4) If there exists a nonzero function $k \in H^{\infty}$ such that $\|\phi-k\|_{\infty} \leqq 1$, by Lemma 3 there exists a nonzero function $f \in H^{1}$ such that $q \bar{q}_{0}=|f| / f$. Hence (3) implies that sing $q_{0} \supset \operatorname{sing} q$, and this contradiction implies that $k=0$ a.e. and so $E(\phi)=\{0\}$. By (3) of Theorem 3, $\rho(\phi)=\mathbb{C} \backslash\{0\}$ or $\rho(\phi)=\mathbb{C}$.

(5) If $q$ is a finite Blaschke product then by (2) of Theorem $2 \rho(\phi)=\mathbb{C}$. Suppose $q$ is not a finite Blaschke product. If $q_{0}$ is a finite Blaschke product then $S_{\bar{q}} \ni z q_{0} h$ for some $h \in H^{1}$ by Theorem 9 in [1] and hence $S_{\phi}$ contains at least two functions. Thus by Theorem $4 \rho(\phi)=\mathbb{C}$. If $q_{0}$ is not a finite Blaschke product then by the hypothesis $E(\phi)=\{0\}$ and $0 \notin \rho(\phi) . E(\phi)=\{0\}$ by the same reason as in (4), and $0 \notin \rho(\phi)$ follows from (3) because $\phi$ is an extremal kernel by the proof of (4). Thus by (3) of Theorem 3 , $\rho(\phi)=\mathscr{C} \backslash\{0\}$.

By (3)-(5) of Theorem 9 we are interested in the case sing $q=\operatorname{sing} q_{0}$.

Corollary 2. Put $q=\prod_{j=1}^{n} q_{j}$ where $q_{j}$ is a non-constant inner function for each $j$. If

$$
\phi=\bar{q} \prod_{j=1}^{m} \frac{q_{j}-a_{j}}{1-\bar{a}_{j} q_{j}}
$$

where $\left|a_{j}\right|<1$ for each $j$ and $m \leqq n$, then $0 \in \rho(\phi)$ and $\phi$ is an extremal kernel. If $m<n$ then $\rho(\phi)=\mathbb{C}$.

Proof.

$$
\prod_{j=1}^{m}\left(q_{j}-a_{j}\right) \in H^{2} \Theta z q H^{2}
$$


because

$$
\begin{aligned}
& \bar{q} \prod_{j=1}^{m}\left(q_{j}-a_{j}\right)=\prod_{j=m+1}^{n} \bar{q}_{j} \prod_{j=1}^{m}\left(1-a_{j} \bar{q}_{j}\right) . \\
& \prod_{j=1}^{m}\left(q_{j}-a_{j}\right)\left(1-\bar{a}_{j} q_{j}\right)^{-1} \times \prod_{j=1}^{m}\left(q_{j}-a_{j}\right)
\end{aligned}
$$

belongs to $H^{2}$ and hence by (1) of Theorem $9,0 \in \rho(\phi)$ and $\phi$ is an extremal kernel. Put

$$
\phi_{0}=\prod_{j=1}^{m} \bar{q}_{j} \prod_{j=1}^{m} \frac{q_{j}-a_{j}}{1-\bar{a}_{j} q_{j}}
$$

then, by what was shown just now, $0 \in \rho\left(\phi_{0}\right)$ and $\phi_{0}$ is an extremal kernel. If $m<n$ then $\phi=\left(\prod_{j=m+1}^{n} \bar{q}_{j}\right) \phi_{0}$ and hence $S_{\phi}$ contains $\left\{\gamma\left(q_{m+1}-a\right)\left(1-\bar{a} q_{m+1}\right) f\right\}$ where $\gamma$ is a positive constant, $f \in S_{\phi_{0}}$ and $a$ is any complex number with $|a| \leqq 1$. By Theorem 4 $\rho(\phi)=\boldsymbol{C}$.

Corollary 3. Let $q$ and $q_{1}$ be nonconstant inner functions with $q \bar{q}_{1} \in H^{\infty}$. Suppose $\left\{z_{j}\right\}_{j=1}^{n}$ is a sequence in the unit disc such that $q_{1}\left(z_{j}\right)=a$ for some complex number a with $|a|<1$. Here $n$ may be infinite or finite. If

$$
\phi=\bar{q} \prod_{j=1}^{n} \frac{\left|z_{j}\right|}{z_{j}} \frac{z-z_{j}}{1-\bar{z}_{j} z}
$$

then $0 \in \rho(\phi)$ and $\phi$ is an extremal kernel. If $q$ is not a scalar multiple of $q_{1}$ then $\rho(\phi)=\mathbb{C}$.

Proof. There exists an inner function $q_{2}$ such that

$$
\frac{q_{1}-q}{1-\bar{a} q_{1}}=q_{2} \times \prod_{j=1}^{n} \frac{\left|z_{j}\right|}{z_{j}} \frac{z-z_{j}}{1-\bar{z}_{j} z} .
$$

By Corollary 2 there exists a function $f$ in $H^{1}$ such that

$$
\bar{q} \frac{q_{1}-a}{1-\bar{a} q_{1}}=\frac{|f|}{f}
$$

and so

$$
\bar{q} \prod_{j=1}^{n} \frac{\left|z_{j}\right|}{z_{j}} \frac{z-z_{j}}{1-\bar{z}_{j} z}=\frac{\left|q_{2} f\right|}{q_{2} f}
$$


Thus $0 \in \rho(\phi)$ and $\phi$ is an extremal kernel. As in the proof of Corollary 2 we show $\rho(\phi)=\mathbb{C}$ if $q$ is not a scalar multiple of $q_{1}$.

Corollary 4. Let $q$ be an inner function, $\phi_{0}(z)=\sum_{j=0}^{n} \alpha_{j} \bar{z}^{j}$ and $\phi=\phi_{0}(q)$.

(1) If $n=1$ then $\rho(\phi)=\mathbb{C}$.

(2) If $n=1, \alpha_{0} \neq 0$ and $\alpha_{1} \neq 0$ then $S_{\phi_{0}}=\left\{(1-\bar{a} z)^{2} /\left\|(1-\bar{a} z)^{2}\right\|_{1}\right\}$ for a nonzero a with $|a| \leqq 1$. However $S_{\phi}$ does not coincide with $\left\{(1-\bar{a} q)^{2} /\left\|(1-\bar{a} q)^{2}\right\|_{1}\right\}$ if $q(0) \neq 0$ or $q(0) \neq$ $1 / \bar{a}+(a-1 / \bar{a}) \alpha$.

(3) For any $n$

$$
\rho(\phi) \supseteq \sum_{j=0}^{n} \alpha_{j} \overline{q(0)}^{j}+\left\{s \in \mathbb{C}:|s|>\left\|\phi+H^{\infty}+C\right\|\right\}
$$

(4) For any $n$, if $q(0)=0$ then $\rho(\phi)=\mathbb{C}$.

Proof. (1) follows from (2) of Corollary 1. (2) It is known that, if $\alpha_{0} \neq 0$ and $\alpha_{1} \neq 0$, then $\alpha_{1}=-a \alpha$ and

$$
\begin{gathered}
S_{\alpha_{0}+\alpha_{1} \bar{z}}=S_{\alpha \bar{z}(z-a / 1-\bar{a} z)}=\left\{(1-\bar{a} z)^{2} /\left\|(1-\bar{a} z)^{2}\right\|_{1}\right\} . \\
\left(\alpha_{0}+\alpha_{1} \bar{q}\right)-\alpha \bar{q} \frac{q-a}{1-\bar{a} q} \in q H^{\infty}
\end{gathered}
$$

but if

$$
q(0) \neq 0, \alpha_{0} q+\alpha_{1}-\alpha \frac{q-a}{1-\bar{a} q} \notin z H^{\infty}
$$

Hence

$$
S_{\alpha_{0}+\alpha_{1} \bar{q}} \neq S_{\alpha \bar{q}(q-a / 1-\bar{a} q)} \ni(1-a q)^{2} /\left\|(1-\bar{a} q)^{2}\right\|_{1} .
$$

(3) follows from (1) of Theorem 3. (4) By (2) of Theorem 2, $S_{\phi_{0}}$ is nonempty. Since there is $k \in z H^{\infty}$ such that $\phi_{0}=\bar{z}^{n+1} k$, by Proposition 8 there exists a finite Blaschke product $b$ of degree at least $n+1$ such that $\psi_{0}=\alpha \bar{z}^{n+1} b$ is the extremal kernel of $\phi_{0}$. Put $\psi=\psi_{0}(q)$ and $\phi=\phi_{0}(q)$; then $\psi$ is the extremal kernel of $\phi$ because $q(0)=0$. By Corollary 2, $0 \in \rho(\phi)$.

\section{Interpolation Blaschke product}

Let $\left\{z_{n}\right\}$ be a sequence of distinct points in the open unit disc. Put

$$
\rho_{n}=\prod_{m ; m \neq n}\left|\frac{z_{n}-z_{m}}{1-\bar{z}_{m} z_{n}}\right|
$$


Let $b$ be a Blaschke product with zeros $\left\{z_{n}\right\}$. We call $b$ an interpolation Blaschke product when $\inf \rho_{n}>0$, that is, $\left\{z_{n}\right\}$ is a uniformly separated sequence.

Proposition 10. Let $b$ be a Blaschke product with zeros $\left\{z_{n}\right\}$ which is the union of $a$ finite number of uniformly separated sequences and let $k \in H^{\infty}$. Suppose $\phi=5 k$ and $\rho_{n}^{-1} k\left(z_{n}\right) \rightarrow 0$ as $n \rightarrow \infty$. Then $\rho(\phi)=\mathbb{C}$.

Proof. By Lemma 3 in [11], $b k \in H^{\infty}+C$ and hence by (2) of Theorem $2, \rho(\phi)=\varnothing$.

Theorem 11. Let $b$ be an interpolation Blaschke product with zeros $\left\{z_{i}\right\}$ and $b_{0} a$ Blaschke product with zeros $\left\{a_{j}\right\}$. Put $\phi=5 b_{0}$. Then $0 \in \rho(\phi)$ and $\phi$ is an extremal kernel if and only if an infinite matrix $\left\{1 / 1-\bar{z}_{i} a_{j}\right\}_{i, j=1}^{\infty}$ has a nontrivial kernel in $\ell^{2}$.

Proof. Since $\left\{z_{i}\right\}$ is a uniformly separated sequence, $\left\{1 / 1-\bar{z}_{i} z\right\}_{i=1}^{\infty}$ is an unconditional basis in $H^{2} \ominus b z H^{2}$ (see [6]). If an infinite matrix $\left\{1 / 1-\bar{z}_{i} a_{j}\right\}_{i, j=1}^{\infty}$ has a nontrivial kernel, then for some $\left\{c_{i}\right\} \in \ell^{2}$

$$
\sum_{i=1}^{\infty} c_{i} \frac{1}{1-\bar{z}_{i} a_{j}}=0 \quad j=1,2, \ldots
$$

Put $f(z)=\sum_{i=1}^{\infty} c_{i}\left(1 / 1-\bar{z}_{i} z\right)$; then $f \in H^{2} \Theta b z H^{2}$ because $\left\{1 / 1-\bar{z}_{i} z\right\}_{i=1}^{\infty}$ is an unconditional basis in $H^{2} \Theta b z H^{2}$. Now $f\left(a_{j}\right)=0$ for $j=1,2, \ldots$. Hence $\bar{b}_{0} f \in H^{2}$. By (1) of Theorem 9, $0 \in \rho(\phi)$ and $\phi$ is an extremal kernel. Conversely if $0 \in \rho(\phi)$ and $\phi$ is an extremal kernel then by (1) of Theorem 9, there exists $f \in H^{2} \Theta b z H^{2}$ such that $b_{0} f \in H^{2}$. Since $\left\{1 / 1-\bar{z}_{i} z\right\}_{i=1}^{\infty}$ is an unconditional basis in $H^{2} \Theta b z H^{2}$,

$$
\begin{gathered}
f=\sum_{i=1}^{\infty} c_{i} \frac{1}{1-\bar{z}_{i} z} \text { and }\left\{c_{i}\right\} \in \ell^{2} . \text { Then } \\
\sum_{i=1}^{\infty} c_{i} \frac{1}{1-\bar{z}_{i} a_{j}}=0 \quad j=1,2, \ldots
\end{gathered}
$$

This proves the theorem.

\section{Applications}

Let $\left\{z_{n}\right\}$ be a Blaschke sequence and let a bounded sequence $\left\{w_{n}\right\}$ be given. If we can find an $f$ in $H^{\infty}$ such that $f\left(z_{n}\right)=w_{n}$ we may assume that $\|f\|_{\infty}$ is minimal. Such an $f$ need not be unique, but K. Øyma gave a sufficient condition for uniqueness. Let $\left\{z_{n}\right\}$ be a uniformly separated sequence in the unit disc and assume $w_{n} \rightarrow 0$. Then there exist a unique $f$ in $H^{\infty}$ of minimal norm such that $f\left(z_{n}\right)=w_{n}$ for all $n$ [12, Theorem 2]. The author [11] gave a sufficient condition for uniqueness in the case of the union of a finite number of uniformly separated sequence $\left\{z_{n}\right\}$, that contains the result of K. Øyma. The 
following theorem gives a solution on this problem in the case of a Blaschke sequence $\left\{z_{n}\right\}$.

Theorem 12. Let $\left\{z_{n}: n=0,1,2, \ldots\right\}$ be a Blaschke sequence with $z_{0}=0$ and $\left\{s, w_{1}, w_{2}, \ldots\right\}$ a bounded sequence. Let $b$ be a Blaschke product with zeros $\left\{z_{1}, z_{2}, \ldots\right\}$. Suppose there exists a function $f$ in $H^{\infty}$ such that $f(0)=0$ and $f\left(z_{j}\right)=w_{j}$ for $j=1,2, \ldots$ If $\rho(\bar{b} f) \ni-s b(0)^{-1}$ then there exists a unique $g$ in $H^{\infty}$ of minimal norm such that $g(0)=s$ and $g\left(z_{j}\right)=w_{j}$ for $j=1,2, \ldots$. This function is a complex constant times an inner function and has analytic continuation across $T \backslash\left\{\overline{z_{n}}\right\}$.

Proof. If $\rho(\bar{b} f) \ni-s b(0)^{-1}$ then $S_{\bar{b} f+s b(0)^{-1}}$ is nonempty and hence there exists a unique function $k \in H^{\infty}$ such that $\left\|\bar{b} f+s b(0)^{-1}+z H^{\infty}\right\|=\left\|\bar{b} f+s b(0)^{-1}+z k\right\|_{\infty}$. This implies that there is a unique function $k$ such that $\left\|f+s b(0)^{-1} b+z b H^{\infty}\right\|=\| f+$ $s b(0)^{-1} b+z b k \|_{\infty}$. Let $g=f+s b(0)^{-1} b+z b k$; then $g(0)=s$ and $g\left(z_{j}\right)=f\left(z_{j}\right)$ for $j=1,2, \ldots$, and it is of minimal norm.

In the theorem above, if $\rho(\bar{b} f)=C$ then $\left\{s, w_{1} w_{2}, \ldots\right\}$ for any $s$ has always a unique minimal interpolating function. Proposition 10 shows that if $b$ is a Blaschke product whose zeros is the union of a finite number of uniformly separated sequence $\left\{z_{n}\right\}$ and if $\rho_{n}^{-1} f\left(z_{n}\right) \rightarrow 0$ then $\rho(\bar{b} f)=\varnothing$.

Let $P$ be the orthogonal projection from $L^{2}$ onto $H^{2}$ and $\phi$ a fixed function in $L^{\infty}$. The Hankel operator with symbol $\phi$ is the operator $H_{\phi}$ from $H^{2}$ to $\left(H^{2}\right)^{\perp}$ is defined by $H_{\phi} f=(1-P)(\phi f), f \in H^{2}$. Now we will study when $H_{\phi}$ has an accessible norm, that is, $\left\|H_{\phi}\right\|^{2}$ is an eigenvalue of $H_{\phi}^{*} H_{\phi}$. Put $\gamma(\phi)=\left\{s \in \mathbb{C}: H_{\phi-s \bar{z}}\right.$ has an accessible norm $\}$.

Theorem 13. For any $\phi \in L^{\infty}$,

$$
\gamma(\phi)=\rho(z \phi)
$$

Proof. For any $f \in z H^{1}, \bar{z} f \in H^{1}$ and $\int f(\phi-s \bar{z}) d \theta / 2 \pi=\int \bar{z} f(z \phi-s) d \theta / 2 \pi$. Since $\left\|H_{\phi-s \bar{z}}\right\|=\left\|T_{z \phi-s}\right\|, \gamma(\phi)=\rho(z \phi)$.

Several characterizations of exposed points of the ball $\left(H^{1}\right)$ are known (cf. [10, Theorem 3], [4, Theorem 8]). Now we will give two more characterizations of such functions. Recall that $K_{\phi}=\left\{k \in H^{\infty}:\|\phi-k\|_{\infty} \leqq 1\right\}$ for $\phi \in L^{\infty}$ (see Section 5).

Proposition 14. Let $\phi=f /|f|$ for some nonzero $f \in H^{1}$ with $\|f\|_{1}=1$. Then $f$ is an exposed point of the ball $\left(H^{1}\right)$ if and only if the interior of $K_{\phi}(0)$ does not contain 0 .

Proof. Lemma 5 implies the part of "only if". Conversely, if $f$ is not an exposed point then by Lemma 6

$$
K_{\phi}(0)=\left\{z:|z-g(0)| \leqq|g(0)|, g \in S_{\phi}\right\}
$$

By Theorem 5.2 in [3, Chapter IV], $\left\{g(0): g \in S_{\phi}\right\}$ contains a disc centred at the origin and the interior of $K_{\phi}(0)$ contains 0 . 
Theorem 15. Let $\phi=f /|f|$ for some nonzero $f \in H^{1}$ with $\|f\|_{1}=1$. Suppose $\left\|\phi+H^{\infty}\right\|$ $=1$ and $\rho(\phi) \neq \varnothing . f$ is an exposed point of the ball $\left(H^{1}\right)$ if and only if the boundary of $\rho(\phi)$ contains 0 .

Proof. Since $\left\|\phi+H^{\infty}\right\|=1$, by (2) of Proposition 7

$$
E(\phi)=\left\{z \in \mathbb{C}:|z-g(0)| \leqq|g(0)|, g \in S_{\bar{\phi}}\right\} .
$$

Hence $E(\phi)$ is not a single point, and by Theorems 3 and 5 ,

$$
\mathbb{C} \backslash E(\phi) \subset \rho(\phi) \subset \mathbb{C} \backslash E(\phi)^{0}
$$

because $\rho(\phi) \neq \mathbb{C}$. The result of the theorem now follows.

\section{REFERENCES}

1. K. deLeeuw and W. Rudin, Extreme points and extreme problems in $H^{1}$, Pacific J. Math. 8 (1958), 467-485.

2. P. Duren, Theory of $H^{p}$ Spaces (Academic Press, New York 1970).

3. J. Garnett, Bounded Analytic Functions (Academic Press, New York, N.Y., 1981).

4. E. Hayashi, The solution sets of $H^{1}$ extremal problem, Proc. Amer. Math. Soc. 93 (1985), 690-696.

5. K. Hoffman, Banach Spaces of Analytic Functions (Prentice Hall, Englewood Cliffs, New Jersey, 1962).

6. S. V. Hrušcev, N. K. Nikol'SkiE and B. S. Pavlov, Unconditional bases of exponentials and of reproducing kernels, in Complex Analysis and Spectral Theory (Lecture Notes in Mathematics 864, Berlin-Heidelberg-New York: Springer 1981), 214-335.

7. P. Koosis, Lectures on $H_{p}$ Spaces (London Math. Society Lecture Notes Series, 40, Cambridge Univ. Press, London and New York).

8. M. Lee and D. Sarason, The spectra of some Toeplitz operators, J. Math. Anal. Appl. 33 (1971), 529-543.

9. T. NAKAZI, Exposed points and extremal problems in $H^{1}, J$. Funct. Anal. 53 (1983), 224-230.

10. T. Nakazı, Exposed points and extremal problems in $H^{1}$, II, Tôhoku Math. J. 37 (1985), 265-269.

11. T. NAKAzl, Notes on interpolation by bounded analytic functions, Canad. Math. Bull. 31 (1988), 404-409.

12. K. Øума, Extremal interpolatory functions in $H^{\infty}$, Proc. Amer. Math. Soc. 64 (1977), 272-276.

Department of Mathematics

FACULTY OF SCIENCE

HOKKAIDO UNIVERSITY

SAPPRO 060

JAPAN 